\title{
High-Resolution Micro-Siting Technique for Large Scale Wind Farm Outside of Japan Using LES Turbulence Model
}

\author{
Takanori Uchida \\ Research Institute for Applied Mechanics, Kyushu University, Kasuga-shi, Japan \\ Email: takanori@riam.kyushu-u.ac.jp
}

How to cite this paper: Uchida, T. (2017) High-Resolution Micro-Siting Technique for Large Scale Wind Farm Outside of Japan Using LES Turbulence Model. Energy and Power Engineering, 9, 802-813. https://doi.org/10.4236/epe.2017.912050

Received: October 17, 2017

Accepted: November 19, 2017

Published: November 22, 2017

Copyright $\odot 2017$ by author and Scientific Research Publishing Inc. This work is licensed under the Creative Commons Attribution International License (CC BY 4.0).

http://creativecommons.org/licenses/by/4.0/

\section{(c) (i) Open Access}

\begin{abstract}
The spatial distribution of wind speed varies greatly over steep complex terrain, thus the selection of an optimal site in such terrain for wind turbine construction requires great care. We have developed a numerical model for simulating unsteady flows called RIAM-COMPACT (Research Institute for Applied Mechanics, Kyushu University, COM putational Prediction of Airflow over Complex Terrain), which is based on the LES (Large-Eddy Simulation) technique. The computational domain of RIAM-COMPACT can be varied from several meters to several kilometers, and the model is able to predict airflow over complex terrain with high accuracy. The present paper discusses the application of RIAM-COMPACT to the micro-siting of wind turbines at sites outside Japan. The results from two case studies will be presented.
\end{abstract}

\section{Keywords}

Large Scale Wind Farm, Complex Terrain, Micro-Siting Technique, LES

\section{Introduction}

We have developed an unsteady and non-linear wind synopsis simulator called RIAM-COMPACT (Research Institute for Applied Mechanics, Kyushu University, Computational Prediction of Airflow over Complex Terrain) in order to simulate the airflow on a microscale, i.e., a few tens of $\mathrm{km}$ or less [1]-[10]. In RIAM-COMPACT, large-eddy simulation (LES) has been adopted for turbulence modeling. LES is a technique in which the structures of relatively large eddies are directly simulated and smaller eddies are modeled using a sub-grid scale model. Other wind-synopsis software which has been developed and is currently available within Japan utilizes models based on Reyolds-averaged Navier-Stokes 
equations because of the computational time requirement. These models are called RANS models and are utilized for simulations of stationary flow fields in which the flow properties remain constant in time [11]. However, computer performance has improved rapidly in recent years, and the constraints imposed by computational time have been drastically reduced. LES models, which solve spatially-averaged Navier-Stokes equations, are able to simulate unsteady wind fields subject to constant changes in flow properties. This aspect of LES models makes them considerably different from RANS models, in which the turbulent flow is temporally (Reynold's) averaged. Furthermore, the number of model parameters to be tuned in LES models is significantly smaller than that in RANS models, thus, LES models excel in versatility. If the characteristics of unsteady wind conditions can be easily predicted by an LES model and the simulation results can be captured visually by an animation or other means, the LES model can serve as an alternative to a costly wind tunnel experiment and can also contribute to the series of investigations conducted prior to the construction of a wind farm (WF).

Recently, the values of the availability factors of wind turbine generators (WTGs) on large WFs constructed on complex terrain have fallen below the values projected originally. In other words, WTGs with notably low power output have been identified, and the issues of both internal and external breakdowns of WTGs have emerged. The major cause of these problems is that slight topographical variations become a source of turbulence (terrain-induced turbulence), and turbulence is generated mechanically (directly) at these topographical variations. Furthermore, with global warming, interannual change in the average wind speed as well as the prevailing wind direction on the WF may become causes of the above-mentioned problems reported at large WFs. In our earlier studies, we performed detailed wind synopsis simulations for existing WFs with the use of elevation data with high resolution, i.e., less than $10 \mathrm{~m}$. Based on the simulation results, we proposed techniques which address issues concerning the relocation of WTGs within a WF such as if the current deployment locations of WTGs should be maintained and if poorly operating WTGs should be relocated or discontinued because of the operating cost. However, our past studies addressed only the influence of the topographical variations on the WTGs and their surroundings and curtailed investigations on the influences of turbulence which is generated upstream of the WF and flows into the WF and of surface roughness on WTGs and their surroundings. The present paper discusses case studies of wind simulations (wind risk evaluation) which were conducted with the RIAM-COMPACT CFD software with high accuracy for sites outside Japan. In addition, a method for acquiring the elevation data (topographical information) for sites outside Japan is also described.

\section{Use of Detailed Terrain Data Generated from ALOS PRISM Data}

In order to accurately model the turbulence over terrain and objects on the sur- 
face in wind simulations, use of terrain data with high precision and accuracy is essential. In the past, detailed terrain maps for planned sites for wind turbine construction outside Japan were often not easily obtainable, and alternative terrain information was needed in such cases. Accordingly, we examine the use in wind simulations of elevation data that are created with high-resolution satellite data for sites outside Japan. Such satellite data are available at a uniform level of quality for worldwide locations. Out of a number of terrain datasets which have been created from satellite images and are available to the public, we use one created from PRISM stereo images. Specifically, the satellite data were produced on a made-to-order basis and sold by the Remote Sensing Technology Center of Japan. PRISM is a visible-spectrum sensor operating on the Earth observation satellite ALOS (Japanese name: DAICHI; launched in 2006 by the Japan Aerospace Exploration Agency (JAXA)) and is able to detect objects on the surface. The elevation data that have been prepared from the PRISM data are called a Digital Surface Model (DSM) and include data on buildings, roads, trees, and geometries of other objects on the surface as well as data on the surface terrain. In contrast, numerous grided elevation datasets such as those provided by the Geographical Survey Institute of Japan, called Digital Elevation Model (DEM) datasets, contain only ground surface elevation data. Therefore, DSM datasets, which allow accurate simulations of turbulence generated by objects on the surface, are more suitable than DEM datasets for use in wind simulations.

The ground resolution of the PRISM DSM data is approximately $10 \mathrm{~m}$ (Figure 1). The horizontal and vertical location accuracies of the data are $20 \mathrm{~m}$ and $40 \mathrm{~m}$, respectively, when no positional corrections are made with ground control points (GCPs). Furthermore, because the relative error of the DSM data is $5 \mathrm{~m}$, the topological error of the terrain and objects on the surface is small, and the distortion of the terrain represented in the DSM data as a whole is small. For the wind synopsis analyses in the present study, the DSM data are extracted for simultion target areas and converted to a format which can be loaded onto RIAM-COMPACT. The geographical coordinate system of the PRISM DSM
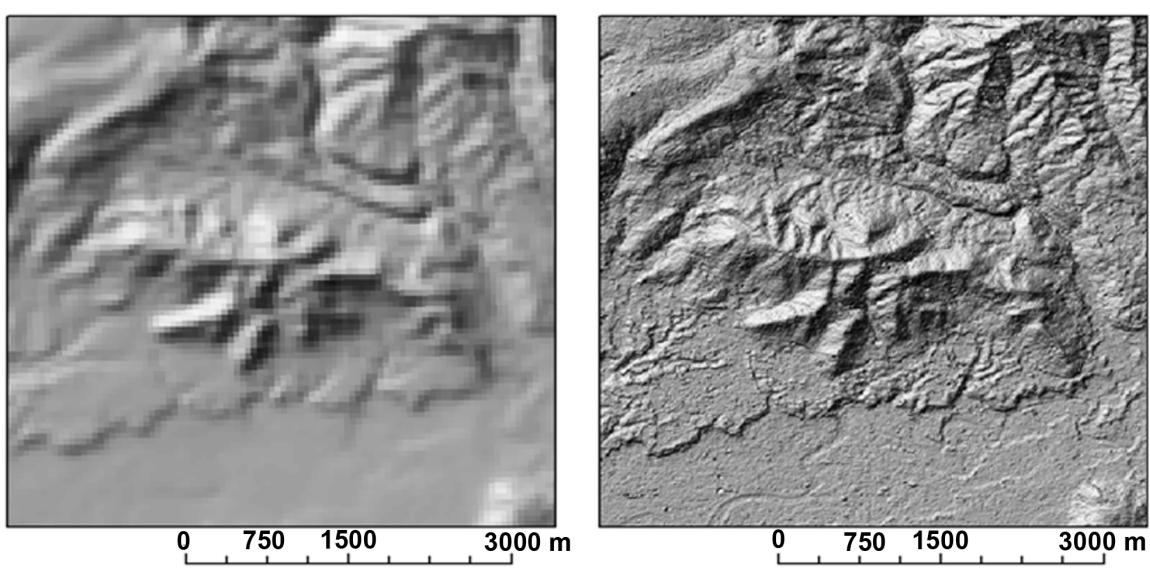

Figure 1. Comparison between SRTM (Shutle Radar Topography Mission) data with 90 $\mathrm{m}$ spatial resolution (left) and ALOS DSM data with $10 \mathrm{~m}$ spatial resolution (right). 
data provided for the present study is ITRF97, in which the terrain data values are assigned to grid points in an equidistant latitude/longitude grid. This coordinate system is almost identical to the latitude/longitude coordinate system that is used in GPS. Therefore, the present DSM data can be used directly in the wind analysis of sites for wind turbine construction without the need for coordinate transformation.

Incidentally, the use of PRISM DSM data is only possible for a potential site for wind power generation if images of the site and its surrounding area have been captured by PRISM from three directions. In some areas outisde Japan, the required data are not available because of the limited number of images in the image library. Because production of terrain data relies on images in the visible spectrum, information about the ground surface cannot be obtained with clouds present. In this case, the elevations of the area of interest cannot be evaluated, and missing value flags for the elevation will be entered in the DSM dataset. Therefore, for subtropical regions, in which clouds are frequently observed throughout the year, the amount of data which can be used for terrain construction tends to be limited. However, the amount of data is expected to grow in the future with continued observation of the Earth's surface by PRISM, and PRISM DSM data will likely become available for more extensive areas than those for which the data are currently available. It is anticipated that the use of highly detailed satellite imagery-based terrain information (Figure 2) for wind power development projects will increase in regions such as developing countries, in which terrain information is highly limited at the present time. The readers are advised to refer to http://global.jaxa.jp/projects/sat/aos//.

\section{Case Studies}

To simulate the airflow over complex terrain with high accuracy and avoid numerical instability, the simulation is performed with RIAM-COMPACT in which collocated grids in a general curvilinear coordinate have been adopted. In the collocated grid system, the velocity components and pressure are defined at
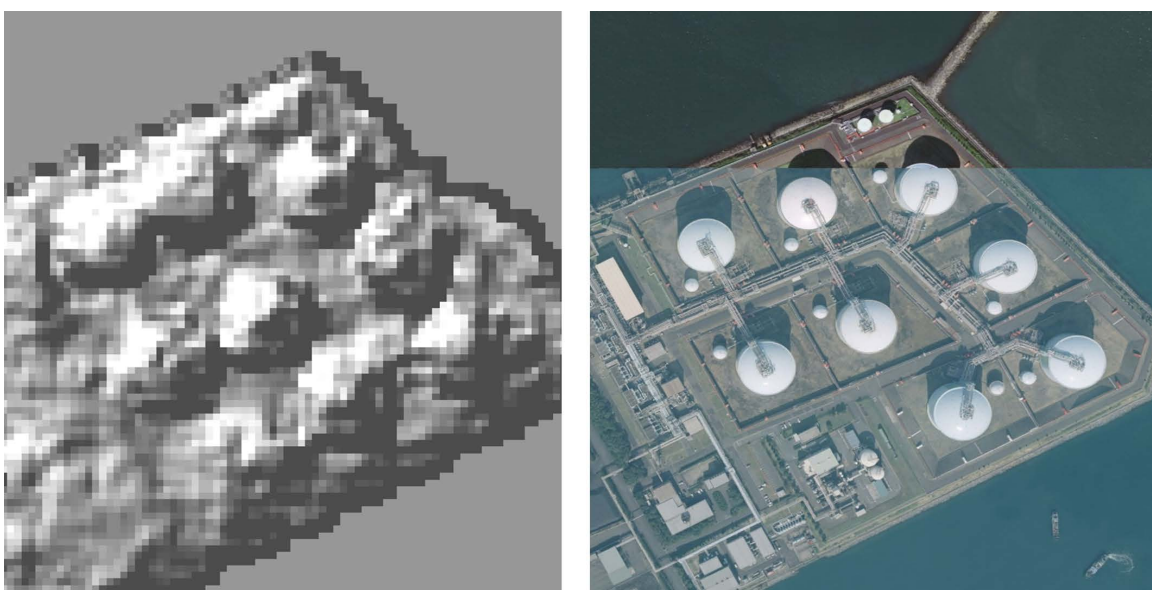

Figure 2. Examples of reconstructed terrain and objects on the surface. 
the cell centers, and variables which result from the covariant velocity components multiplied by the Jacobian are defined at the cell faces. As for the computational technique, the finite-difference method is adopted, and LES is used for the turbulence model. In LES, a spatial filter is applied on the flow field to separate eddies of various scales into grid scale (GS) components, which are larger than the computational grids, and subgrid scale (SGS) components, which are smaller than the computational grids. Large-scale eddies, i.e. the GS components of turbulence eddies, are numerically simulated directly without relying on the use of a physically simplified model. The main effect of small-scale eddies, i.e. the SGS components, is to dissipate energy, and this dissipation is modeled based on the physical considerations of the SGS stress. For the governing equations of the flow, a spatially-filtered continuity equation for incompressible fluid and a spatially filtered version of the Navier-Stokes Equation are used. Because the present study is on airflow prediction in high wind conditions, the effect of the temperature stratification which is generally present in the atmosphere is neglected. The computational algorithm and the time marching method are based on a fractional-step (FS) method [12] and the Euler explicit method, respectively. The Poisson's equation for pressure is solved by the successive over-relaxation (SOR) method. For discretization of all the spatial terms except for the convective term, a second-order central difference scheme is applied. For the convective term, a third-order upwind difference scheme is applied. Generally, the third-order upwind difference equation consists of a fourth-order central difference term and a numerical diffusion term which takes the form of a fourth-order derivative. For the fourth-order central differencing, an interpolation technique based on 4-point differencing and 4-point interpolation by Kajishima [13] is used in the present study. In the weighting of the numerical diffusion term of the third-order upwind differencing, $\alpha=3.0$ is commonly applied in the Kawamura-Kuwahara Scheme [14]. However, $\alpha$ is set to 0.5 in the present study to minimize the influence of numerical diffusion. For LES SGS modeling, the commonly used Smagorinsky model [15] is adopted. A wall-damping function is also used with a model coefficient of 0.1 .

Regarding the boundary conditions, a slip condition is applied at both the upper and side boundaries. Specifically, for the upper boundary, the gradient of the horizontal wind speed components $(u, v)$ in the vertical direction $(\mathrm{z})$ and the vertical wind velocity component $(w)$ are all set to zero. For the side boundaries, the gradients of the streamwise and vertical wind velocity components $(u, w)$ in the spanwise direction $(y)$ and the spanwise wind velocity component $(v)$ are all set to zero. For the outflow boundary and the ground, a convective outflow condition and a non-slip condition are imposed, respectively. At the inflow boundary, a vertical profile of the horizontal streamwise wind speed, $U_{\text {in }}$, is given using a $1 / 7$ power law.

\subsection{China Wind Power Generation Project Site}

First, a case study for wind synopsis evaluation is illustrated for a planned site 
for wind power generation in the China Wind Power Generation Project [12]. The development scale for the present project is $50,000 \mathrm{~kW}(2000 \mathrm{~kW} /$ wind turbine $\times 25$ wind turbines). The planned site for wind power generation is at an elevation of approximately $1700 \mathrm{~m}$, and part of the surrounding terrain is characterized as complex terrain which includes valleys. For the present project, wind synopsis simulations are performed for 16 wind directions using RIAM-COMPACT, in order to evaluate wind conditions for the wind farm area and at the individual wind turbines. The numerical simulations involved approximately one million grid points in a domain which includes the planned site for wind turbine deployment as shown in Figure 3.

Recently, the capacity factors of some wind farms within Japan have fallen below their initial projections, and the significant reduction in power output at these wind farms has become a significant concern. Turbulence which is generated locally by small undulations near wind turbines (terrain-induced turbulence) is commonly considered to be the cause of the low capacity factors. Accordingly, for the present project, the wind synopsis evaluation is made with a focus on the influence of the terrain-induced turbulence on wind power generation. Subsequently, some of the major analyses and results from the numerical wind synopsis evaluation will be discussed. First, the existence of the influence of terrain-induced turbulence on the flow around the wind turbines is visually examined. For the visual examination, an animation is created so that air parcels with large terrain-induced wind velocity fluctuations can be tracked.

Figure 4 shows the wind velocity vectors (instantaneous field) at the wind turbine hub heights. Furthermore, a contour plot of the fluctuating streamwise velocity from the wind field in Figure $4,\left|u^{\prime}\right|(=\mid u-$ Uave $\mid)$, is illustrated in

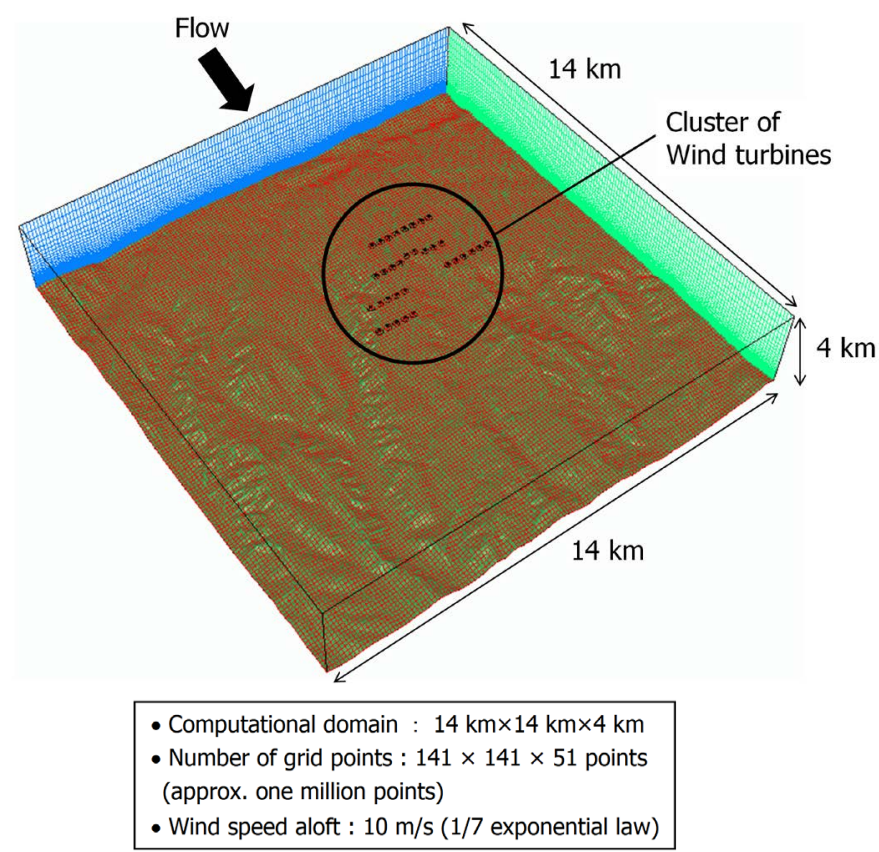

Figure 3. Birds-eye view of the computational domain. 


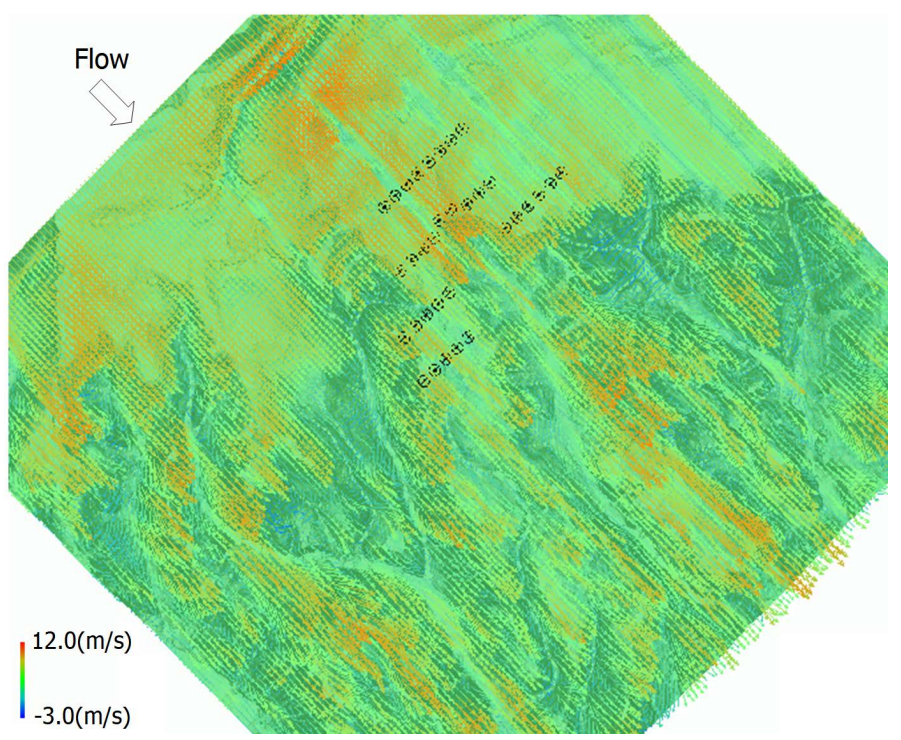

Figure 4. Wind vectors at the wind turbine hub-heights $\left(\mathrm{z}^{*}=65 \mathrm{~m}\right)$.

Figure 5. Because turbulent flow conditions are hard to identify in these still images, spatial and temporal variation in the terrain-induced turbulence has been confirmed with the use of the animation described earlier. The temporal change in the fluctuating streamwise wind velocity component of the terrain-induced turbulence as described in this case study can be investigated as a result of the non-stationary wind synopsis simulation. Moreover, our wind synopsis evaluation which is based on RIAM-COMPACT allows visualization of turbulence as well as complex wind flows, and thus enables wind turbine deployment planning which takes into account the three-dimensional airflow conditions.

Second, a statistical analysis is performed on the simulated wind data. Figure 6 and Figure 7 show the vertical profile of the mean normalized horizontal wind speed and that of the streamwise turbulence intensity, respectively, from four of the wind turbine deployment locations at the China Wind Power Generation Project Site. The vertical axis indicates the height above the ground in full scale, $z^{\star}(m)$. The horizontal axes in Figure 6 and Figure 7 indicate the non-dimensional wind speed and the turbulence intensity, respectively, which have been normalized by the inflow wind velocity, $U_{\text {in }}$ The two figures also include an illustration of the rotor diameter. As part of the evaluation procedure for determining the deployment location of a wind turbine, essential criteria include that neither the horizontal wind speed shear nor the wind speed deficit is large in the vertical profile of the horizontal wind speed. The evaluation procedure also examines the vertical profile of turbulence intensity to determine if the values of turbulence intensity exceed a unique reference value. When the wind turbulence intensity is less than the reference value, the power output from the wind turbine does not decline significantly. The validity of the reference value has been confirmed using a large number of wind synopsis diagnostics for wind farms from our past research. Evaluations of the vertical profiles of the mean horizontal wind speed 


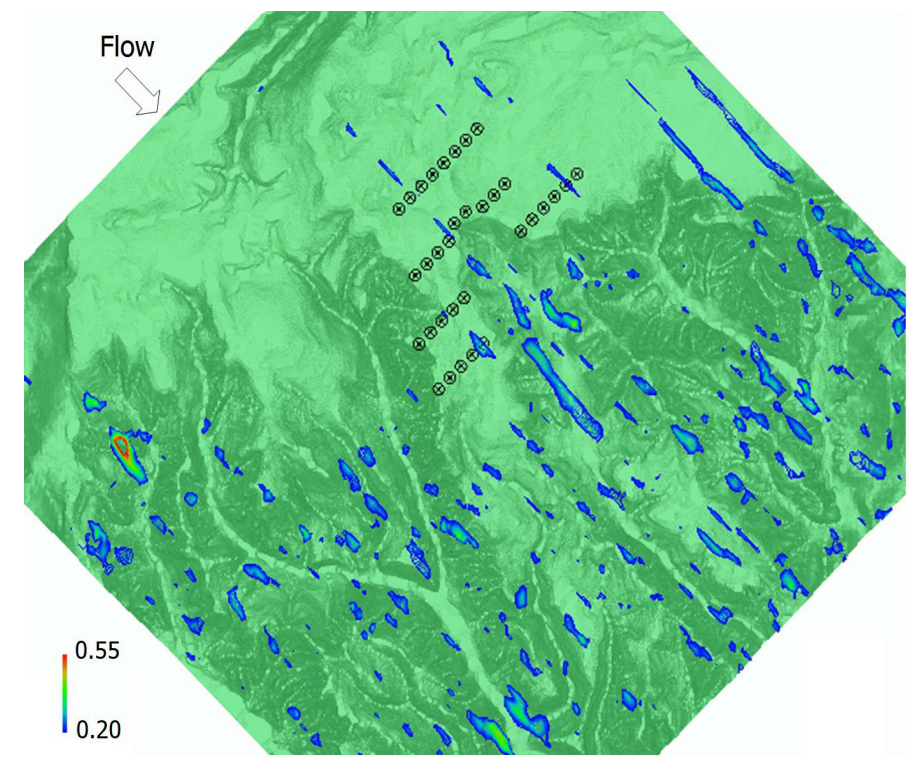

Figure 5. Distribution of the fluctuating streamwise wind velocity component at the wind turbine hub-heights $\left(\mathrm{z}^{\star}=65 \mathrm{~m}\right)$.
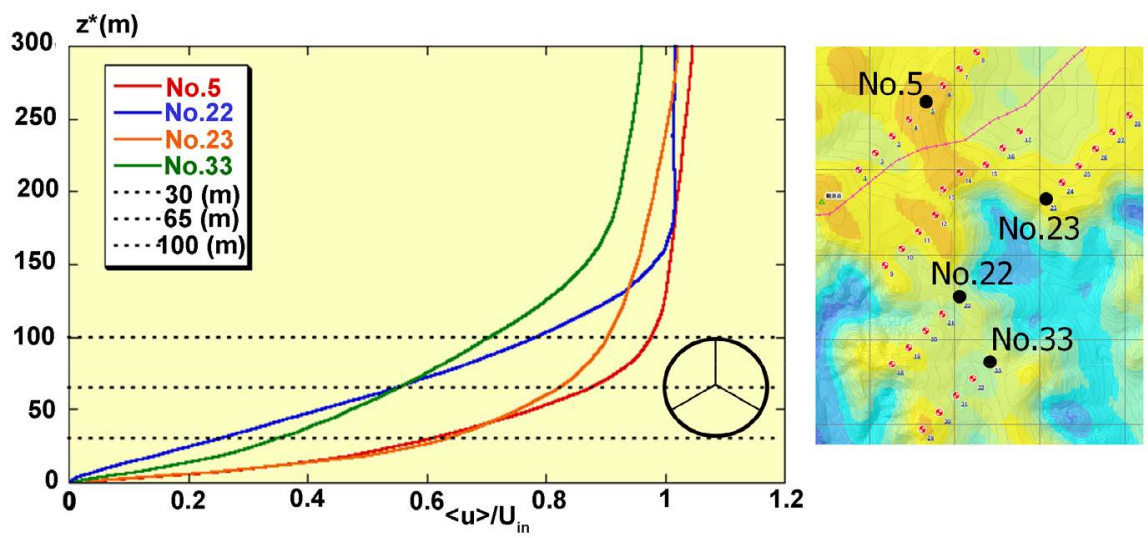

Figure 6. Vertical profiles of the mean horizontal wind speed normalized by the inflow velocity.
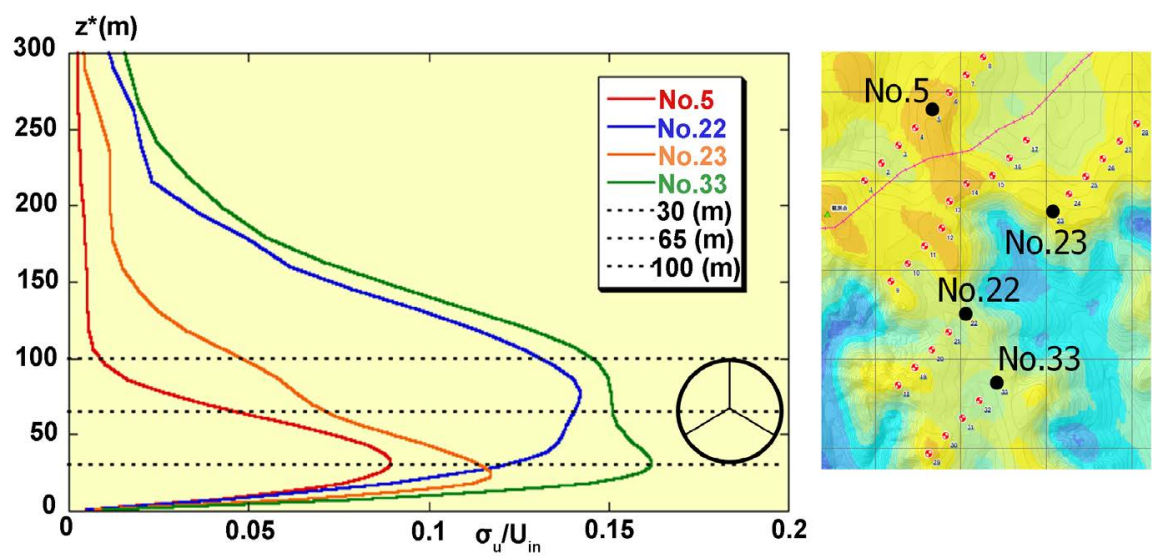

Figure 7. Vertical profiles of streamwise turbulence intensity normalized by the inflow velocity. 
and turbulence intensity enable wind load mitigation on wind turbines, which can also lead to a reduction in the frequency of wind turbine malfunctions.

For the present case study, inter-comparisons of turbulence statistics (vertical profiles of the mean horizontal wind speed and streamwise turbulence intensity) among some of the planned wind turbines were illustrated. Evaluations of the turbulence statistics suggested that stable operations of most of the examined wind turbines could be expected. The investigated wind turbine arrangement was one of the arrangements under consideration, and was not the final arrangement adopted for the site.

\subsection{Palm Springs Wind Power Generation Site}

In this section, a case study of wind evaluation for a large-scale wind farm in the Palm Springs area in California, U.S.A. is discussed. As described earlier, the acquisition of elevation data (terrain information) is necessary for conducting wind synopsis simulations for sites outside as well as within Japan. Currently, while our research group mainly uses the above-mentioned terrain data based on data from the Earth observation satellite ALOS, we also use Space Shuttle SRTM terrain data together with the ALOS terrain data. Accordingly, a case study that uses SRTM and ALOS terrain data will be described below.

The Shuttle Rader Topography Mission (SRTM) is an effort to generate detailed three dimensional topographic data of the entire globe using an on-board radar. At present, there are two three-dimensional SRTM topographic datasets available to the public: SRTM-1 and SRTM-3. The SRTM-1 data are available only for the United States, and their resolution is 1 arc second (approximately 30 $\mathrm{m}$ ). The resolution of the SRTM-3 data is 3 arc seconds (approximately $90 \mathrm{~m}$ ), and the data are available for the entire globe. Readers are advised to refer to https://www.usgs.gov/ for details of the two datasets. In the present study, the computational mesh is generated using SRTM-1 data.

For analysis, a computational domain is defined with a size of $20 \mathrm{~km} \times 10 \mathrm{~km}$ $\times 7 \mathrm{~km}$ in the streamwise (x), spanwise (y), and vertical (z) directions, respectively. The number of computational grid points is $201(\mathrm{x}) \times 101(\mathrm{y}) \times 41(\mathrm{z})$. The other settings used for the analysis are identical to those used for the China Wind Power Generation Project site. Figure 8 shows wind velocity vectors for the prevailing wind direction at the Palm Springs site. The wind velocity vectors displayed in this figure are from the heights which correspond approximately to the hub-heights. Mountain ranges extend in the east-west direction on both the north and south ends of the present computational domain, and the valley between the mountain ranges forms a wind path (see Figure 8). Within this valley, multiple wind turbines have been constructed in a concentrated manner. The locations (latitude and longitude information) of some representative wind turbines out of the thousands within the valley are determined from visual inspections using Google Earth, and the location information is included in the simulation. Figure 8 suggests that no large spatial variations exist in the wind 


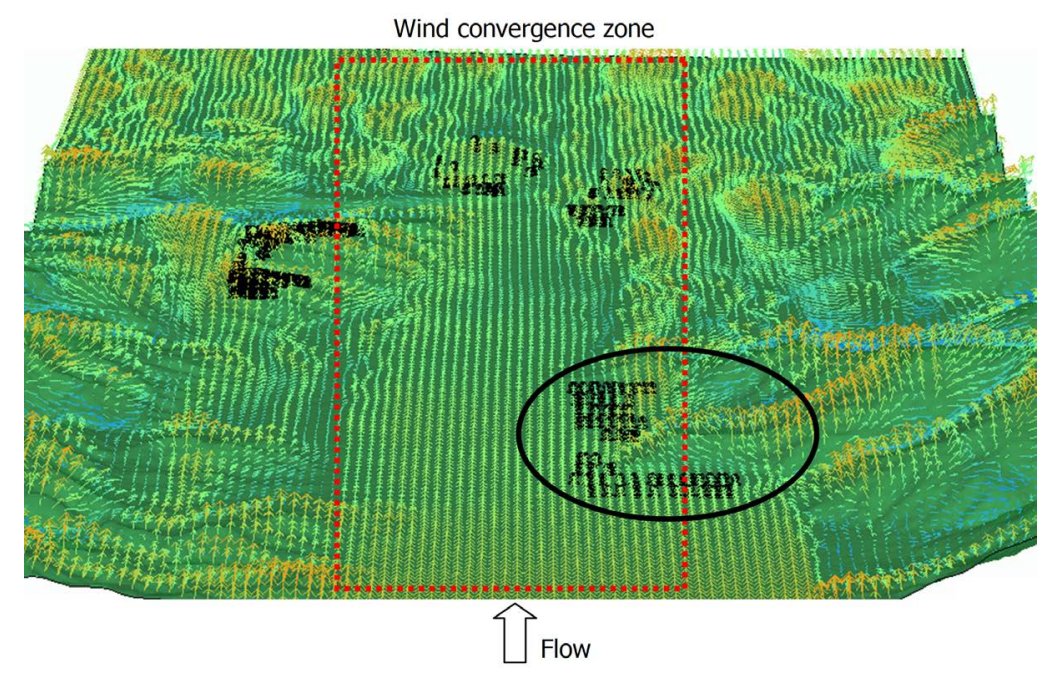

Figure 8. Distribution of wind velocity vectors under the prevailing wind direction (westerly).

direction in the valley in the middle of the computational domain (the area marked by dotted lines), thus the wind in this area flows steadily with little temporal fluctuation. It can be speculated that this area corresponds to a zone of wind convergence. On the other hand, complex wind patterns exist on the north and south sides of the computational domain outside of the region indicated by dotted lines.

Figure 9 illustrates a magnified view of the area indicated by the black circle in Figure 8 and Figure 10 is a photograph of the same area. An examination of Figure 9 reveals that the topography situated adjacent to the wind turbines affects the wind field in the area marked by dotted lines, that is, the wind speed increases locally at the locations where the wind flows around the topography, and the wind turbines in the area indicated by dotted lines are likely influenced by the wind with increased speed. Efficient power generation can be anticipated from wind turbines in such conditions.

\section{Conclusions}

For wind turbine deployment planning, detailed investigations which include wind synopsis evaluations are necessary. Wind synopsis evaluations require a non-stationary wind synopsis technique and also a detailed terrain data construction technique which takes into account the latest terrain conditions. As for the visualization of the wind field, conventional wind mapping alone in which the stationary wind velocity distribution is visualized for the horizontal cross-section at the hub-heights is insufficient. In particular, the increasing size of wind turbine generators calls for evaluations of the wind velocity distribution along vertical cross-sections, thus, displays of vertical profiles of the turbulence intensity as well as those of the mean horizontal wind speed will likely become increasingly relevant from this time forward. 


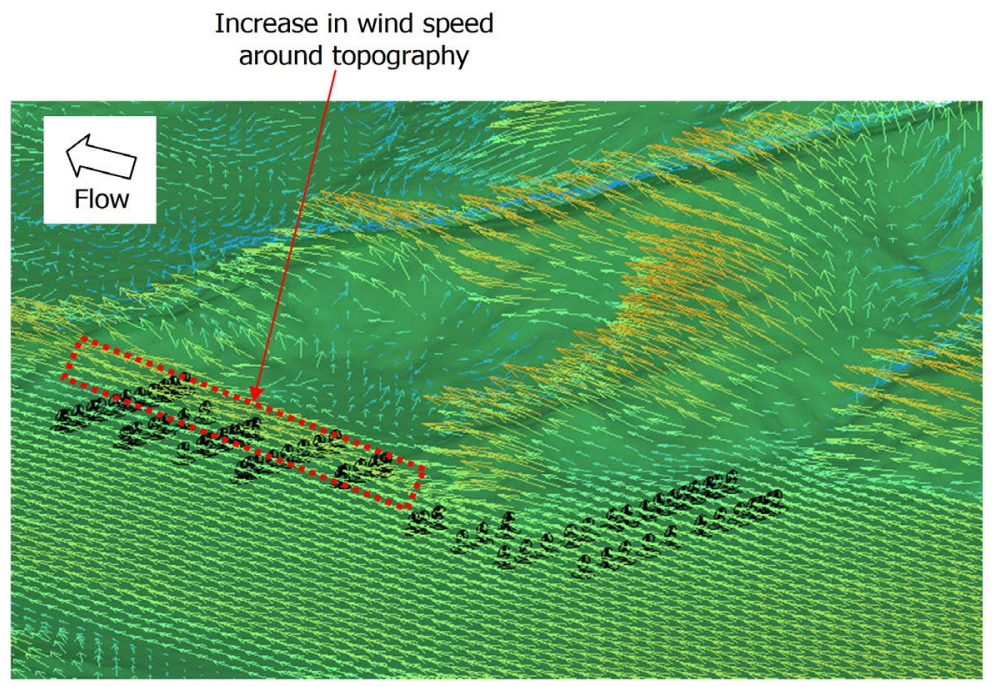

Figure 9. Enlarged view of the area indicated by the black circle in Figure 8.

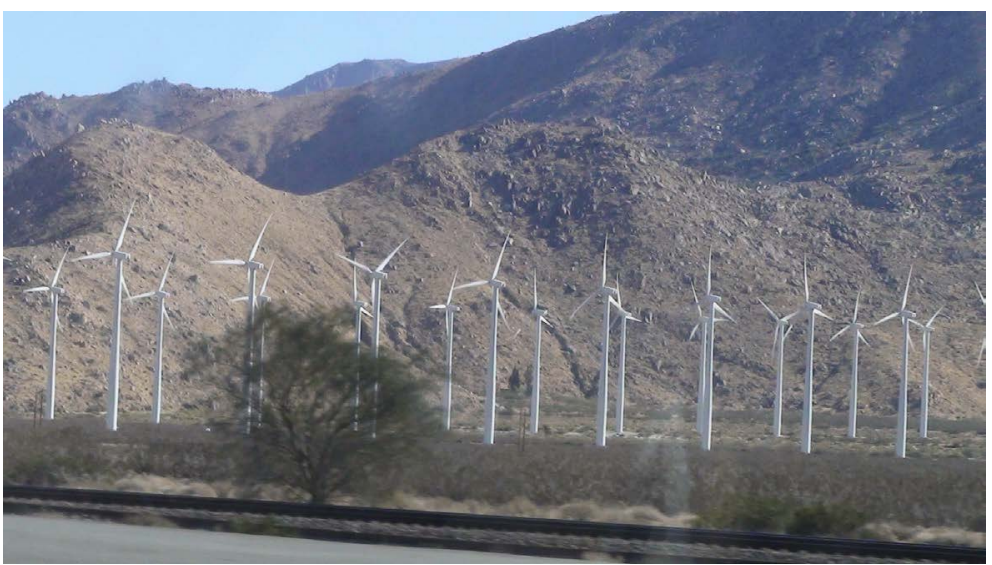

Figure 10. Photograph of the wind turbine site indicated by the black circle in Figure 8 (photograph by the author).

Further detailed investigations that use numerical simulations, wind-tunnel experiments, and field observation data are necessary for identifying the level of wind velocity fluctuation, above which there will be adverse effects on the power generation performance. These investigations will be addressed in our future research.

\section{Acknowledgements}

This work was supported by JSPS KAKENHI Grant Number 17H02053. Also, a part of the present research was supported by Mr. Ryo ARAYA (Environmental GIS Laboratory Co., Ltd.) and Mr. Yasushi KAWASHIMA (West Japan Engineering Consultants, Inc.). The author expresses appreciation to them.

\section{References}

[1] Uchida, T. and Ohya, Y. (2008) Micro-Siting Technique for Wind Turbine Generators by Using Large-Eddy Simulation. Journal of Wind Engineering \& Industrial 
Aerodynamics, 96, 2121-2138. https://doi.org/10.1016/j.jweia.2008.02.047

[2] Watanabe, K., Ohya, Y., Uchida, T. and Nagai, T. (2017) Numerical Prediction and Field Verification Test of Wind-Power Generation Potential in Nearshore Area Using a Moored Floating Platform. Journal of Flow Control, Measurement \& Visualization, 5, 21-35. https://doi.org/10.4236/jfcmv.2017.52002

[3] Watanabe, F. and Uchida, T. (2015) Micro-Siting of Wind Turbine in Complex Terrain: Simplified Fatigue Life Prediction of Main Bearing in Direct Drive Wind Turbines. Wind Engineering, 39, 49-368.

[4] Uchida, T., Ohya, Y. and Sugitani, K. (2011) Comparisons between the Wake of a Wind Turbine Generator Operated at Optimal Tip Speed Ratio and the Wake of a Stationary Disk. Modelling and Simulation in Engineering, 2011, Article ID: 749421. http://dx.doi.org/10.1155/2011/749421

[5] Uchida, T., Maruyama, T. and Ohya, Y. (2011) New Evaluation Technique for WTG Design Wind Speed Using a CFD-Model-Based Unsteady Flow Simulation with Wind Direction Changes. Modelling and Simulation in Engineering, 2011, Article ID: 941870. http://dx.doi.org/10.1155/2011/941870

[6] Uchida, T. and Ohya, Y. (2011) Latest Developments in Numerical Wind Synopsis Prediction Using the RIAM-COMPACT CFD Model-Design Wind Speed Evaluation and Wind Risk (Terrain-Induced Turbulence) Diagnostics in Japan. Energies, 4, 458-474. https://doi.org/10.3390/en4030458

[7] Uchida, T. and Ohya, Y. (2008) Verification of the Prediction Accuracy of Annual Energy Output at Noma Wind Park by the Non-Stationary and Non-Linear Wind Synopsis Simulator, RIAM-COMPACT. Journal of Fluid Science and Technology, 3, 344-358. https://doi.org/10.1299/jfst.3.344

[8] Uchida, T. and Ohya, Y. (2006) Application of LES Technique to Diagnosis of Wind Farm by Using High Resolution Elevation Data. JSME International Journal (Environmental Flows), Series B, 49, 567-575.

[9] Uchida, T. and Ohya, Y. (2003) Large-Eddy Simulation of Turbulent Airflow over Complex Terrain. Journal of Wind Engineering \& Industrial Aerodynamics, 91, 219-229. https://doi.org/10.1016/S0167-6105(02)00347-1

[10] Uchida, T. and Ohya, Y. (1999) Numerical Simulation of Atmospheric Flow over Complex Terrain. Journal of Wind Engineering \& Industrial Aerodynamics, 81, 283-293. https://doi.org/10.1016/S0167-6105(99)00024-0

[11] Sumner, J., Watters, C.S. and Masson, C. (2010) Review: CFD in Wind Energy: The Virtual, Multiscale Wind Tunnel. Energies, 3, 989-1013. https://doi.org/10.3390/en3050989

[12] Kim, J. and Moin, P. (1985) Application of a Fractional-Step Method to Incompressible Navier-Stokes Equations. Journal of Computational Physics, 59, 308-323. https://doi.org/10.1016/0021-9991(85)90148-2

[13] Kajishima, T. (1999) Finite-Difference Method for Convective Terms Using Non-Uniform Grid. Transactions of the Japan Society of Mechanical Engineers, Part B, 65, 1607-1612.

[14] Kawamura, T., Takami, H. and Kuwahara, K. (1986) Computation of High Reynolds Number Flow around a Circular Cylinder with Surface Roughness. Fluid Dynamics Research, 1, 145-162. https://doi.org/10.1016/0169-5983(86)90014-6

[15] Smagorinsky, J. (1963) General Circulation Experiments with the Primitive. Equations, Monthly Weather Review, 91, 99-164. https://doi.org/10.1175/1520-0493(1963)091<0099:GCEWTP >2.3.CO;2 